\title{
La planificación estratégica en el sistema de medición de gestión en la industria petrolera venezolana
}

\section{Strategic planning in the management measurement system in venezuelan oil industry}

Yajaira Alvarado

yaalv26@gmail.com

https://orcid.org/0000-0001-5221-2302

Universidad del Zulia, Maracaibo - Venezuela

\section{Jelvis Chirinos}

jelchirinos@gmail.com

https://orcid.org/0000-0002-8041-2616

Universidad del Zulia, Maracaibo - Venezuela

Artículo recibido en enero 2021 / Arbitrado en marzo 2021 / Aceptado en abril 2021 / Publicado en julio 2021

La investigación tuvo como objetivo identificar los elementos de la planificación estratégica presentes en el sistema de medición de gestión en las gerencias de planificación y gestión de la industria petrolera venezolana en la región occidente. Por su alcance, se consideró una investigación descriptiva, con diseño no experimental, transeccional y de campo. La población la conformaron los gerentes y líderes de las gerencias de planificación y gestión. Para la recolección de datos se aplicó como técnica la encuesta, con un cuestionario de 28 ítems, validado mediante el juicio de expertos. La confiabilidad se calculó a través del coeficiente Alfa de Cronbach, cuyo resultado fue de 0,91. El análisis de los datos se realizó mediante la estadística descriptiva, a través del estudio de las medias aritméticas. Se concluyó alta presencia de los elementos de la planificación estratégica como un proceso fundamental que nutre de información el sistema de medición de gestión.

Palabras clave: Gestión; Planificación estratégica; Proceso; Sistema de medición; Información

ABSTRACT

The research aimed to identify the strategic planning elements present in the management measurement system in the planning and management departments of the Venezuelan oil industry in the western region of the country. Due to its scope, it was considered a descriptive investigation, with a non-experimental, transectional, and field design. The population was made up of the managers and leaders of the planning and management departments. For the data collection, the survey was applied as a technique, with a 28item questionnaire, validated by expert judgment. Reliability was calculated through Cronbach's alpha coefficient whose result was 0.91 . The data analysis was performed using descriptive statistics, through the study of arithmetic means. The high presence of the elements of strategic planning was concluded as a fundamental process that nourishes the management measurement system with information.

Keywords: Management; Strategic planning; Process; Measurement system; Information 


\section{INTRODUCCIÓN}

A través de los resultados de numerosas investigaciones, a nivel mundial, se puede afirmar que en la actualidad y, con miras hacia el futuro, solo aquellas empresas que interiorizan un componente ético sobreviven y prosperan. Esta afirmación está asociada a la percepción de sus clientes, el nivel de compromiso o sentido de pertenencia de su personal, su reputación ante la competencia y sociedad en general.

En este sentido, a medida que la gerencia detecte la necesidad de mejorar sus resultados operacionales y financieros, éstas deberán basarse en el diseño de técnicas innovadoras que le permitan obtener mejoras progresivas a corto, mediano y largo plazo, mediante estrategias que conlleven al cumplimiento de todos los objetivos, desde el más general hasta los más específicos, controlando cada una de estas técnicas a través de indicadores que permitan el seguimiento al desempeño organizacional.

De esta manera, en el contexto empresarial surge la concepción del sistema de medición de gestión como una herramienta competitiva que soporta a las empresas para optimizar sus recursos generando valor en sus procesos, cumpliendo con las entregas al consumidor final con altos niveles de satisfacción (Serna, 2008). Para ello las empresas deben tener claro lo que implica la medición de su gestión. La primera establece un plano operativo de la acción (control), mientras que la segunda es el proceso gerencial estratégico (gestión).

Desde lo estratégico, afirma Serna (2008), se procede partiendo del establecimiento de un conjunto de elementos de análisis que justifiquen la necesidad de enfocar los sistemas de control en función de la estrategia, así como de la estructura de la organización, otorgándole al sistema de información elementos de análisis tanto cuantitativos como cualitativos, elementos financieros y no financieros, resumidos todos en los llamados factores formales y no formales del control.
Desde el ambito operacional, según Francés (2006), es necesario el analisis de los sistemas de gestión, los cuales constituyen un elemento fundamental para lograr la eficacia, ya que contribuyen al mejoramiento continuo de los procesos, reducción del desperdicio, mejora en la comunicación y moral del personal, fortalecimiento en la relacion cliente-proveedor, direccionamiento del trabajo, prevención de fallas, con el objeto de consolidar el trabajo de la empresa con una visión enfocada a garantizar la calidad del producto o servicio y la satisfacción del cliente tanto interno como externo. Es importante destacar que debe existir un sistema de alimentación y retroalimentación de información, eficiente y eficaz para la toma de decisiones generadas del sistema de control de gestión sistémico y estratégico.

En este marco de referencia, Serna (2008) define el sistema de medición de gestión como el conjunto de indicadores medibles, derivados del plan estratégico, que permiten evaluar con índices el alineamiento entre estrategias, objetivos, acciones y resultados, por tanto, determinar el desempeño de la organización frente a su direccionamiento estratégico. De manera más específica, para Beltrán (2010), el sistema de medición de gestión permite la interacción entre las etapas del ciclo administrativo: planeación, organización, dirección y control; sustentando los planes de mejoramiento, de donde se debe obtener como resultado altos niveles de calidad, eficiencia y eficacia.

Ahora bien, en América Latina, según López (2008), el control de la gestión se prepara para prever los impactos gerenciales, así como los acondicionamientos económicos, políticos, sociales y tecnológicos, producto de la intervención o las variaciones en escenarios diversos. Las empresas bajo estos escenarios, en su afán de responder a los impactos, y sumados a las exigencias tanto del mercado como las del consumidor, procuran desarrollar productos/servicios de calidad como opción para ser competitivas. 
En concordancia con lo expuesto, Venezuela no escapa de las exigencias planteadas a nivel mundial para el crecimiento y fortalecimiento de sus organizaciones empresariales, donde las industrias puedan ser capaces de sobrepasar las expectativas del cliente, brindando calidad y diversidad, y del mismo modo poseer la capacidad productiva $y$ organizacional para hacer intercambios comerciales con el mercado extranjero. Atendiendo a esto, se hace necesario un estudio más profundo de la situación de las industrias, a fin de determinar la existencia y modos de implantación de sistemas de medición de gestión, tanto en lo estratégico como en lo operativo, con el objetivo de poder realizar un máximo aprovechamiento de las oportunidades que le brinda el entorno y de las fortalezas que las industrias posean.

Planteadas las ideas anteriores, sobre el sistema de medición de gestión, importancia, aplicabilidad y aspectos a considerar en su estudio, a continuación, se hace referencia al sector que representó la inquietud de los investigadores. En este contexto, Terán (2011) afirma que las organizaciones petroleras deben ser competitivas $\mathrm{y}$ ofrecer una entrega oportuna de productos de calidad. Este nuevo entorno de exigencias ha comprometido a gerentes y a ingenieros a optimizar todos sus procesos.

Bajo tales consideraciones, el sistema de medición de gestión representa una función clave para apoyar a las operaciones, propiamente dichas, y contribuir al logro de los objetivos de la organización. Estas organizaciones, han concentrado esfuerzos en el aumento de sus capacidades, evolucionando hacia la mejora de su eficiencia, que lleva a las mismas a la obtención de sus metas. En esta perspectiva, al sector petrolero a nivel mundial se le confiere el carácter de base energética fundamental de la sociedad moderna, con base en el petróleo como fuente de energía con múltiple utilización, por ello la industria petrolera es considerada una de las más importantes.
Adentrando un poco más en el desarrollo del presente estudio, con base a Terán (2011) es necesario acotar que, desde el último tercio del siglo XIX, prácticamente todas las actividades económicas se sustentan en el petróleo como fuente energética, representando alrededor del $40 \%$ de la necesidad mundial. Dándole la importancia a la complejidad y aumento del mercado a través de un bien, con características intrínsecas, vinculadas con el acontecer de todo el planeta, como la industria petrolera.

En este punto, la industria petrolera en Venezuela no debería quedar relegada del resto del mundo. En este sentido, Petróleos de Venezuela Sociedad Anónima (PDVSA), es la industria del país en el sector de hidrocarburos, cuya misión es diseñar estrategias que permitan la recuperación eficiente y rentable de la reserva del mismo, mediante la elaboración de planes, explotación, promoviendo el mejoramiento continuo en los procesos, garantizando el manejo de la gestión con sentido del negocio, basados en el desarrollo del personal y tecnología, con el mayor grado de seguridad, protección ambiental, calidad, flexibilidad, dirigidos a la máxima satisfacción de sus clientes, en la búsqueda permanente del éxito, satisfacer las necesidades energéticas de la sociedad.

Asimismo, PDVSA es una corporación mundial de energía, propiedad del Estado venezolano, que se encarga del desarrollo de la industria petrolera, petroquímica y carbonífera. Su función es planificar, coordinar, supervisar y controlar las actividades de sus empresas operadoras más las filiales, tanto en Venezuela como en el exterior.

En su estructura organizacional se ubican las gerencias de planificación y gestión, encargadas de establecer planes estratégicos que contribuyan a la ejecución a nivel operacional de las actividades de perforación, rehabilitación, servicios realizados con taladros y trabajos menores ejecutados por equipos de subsuelo; así mismo genera con frecuencia mensual el informe de indicadores de gestión 
denominado rendición de cuentas, permitiendo el análisis de ejecución real versus la planificada del año fiscal.

Estas gerencias emplean un proceso de medición de gestión normalizado, basado en las mejores prácticas, guiadas por normas y procedimientos creadas por ellas mismas, las cuales contienen lineamientos prácticos para la efectiva ejecución de la medición y control de su gestión de manera sistemática u ordenada, tratando de no omitir ningún paso importante.

Garantizando de esta manera un alto grado de confianza en el éxito y en el cumplimiento de los requisitos solicitados por PDVSA. Éste es el enfoque de las gerencias de planificación y gestión de la industria petrolera, utilizado a fin de alcanzar las más altas metas de eficiencia en un mundo totalmente cambiante como el actual.

Ahora bien, en estas gerencias se ha venido observando un mayor reconocimiento, por parte del estrato gerencial, de la importancia que tiene lograr una gestión efectiva para alcanzar un control eficiente de sus procesos. Aún cuando, según la percepción de los investigadores, por ser parte del personal que labora en la misma, existen deficiencias en la visión global de los procesos de medición, lo cual ha generado debilidades en todo el proceso de gestión, ya que la información no fluye correctamente.

A este respecto, en entrevistas realizadas a los gerentes de estas dependencias de la industria petrolera occidente, señalaron la falta de herramientas gerenciales existentes parala medición de los procesos que desarrollan, repercutiendo de forma negativa en su operacionalidad, debido a que el sistema de medición de gestión no ha sido aplicado de manera adecuada como herramienta de evaluación progresiva, independiente y sistemática, en la medición del desempeño en las actividades, costos, tiempo y calidad.

En esta perspectiva, según la experiencia de los investigadores, en las actividades desempeñadas en las gerencias de planificación y gestión se evidencia la falta de monitoreo o control efectivo, que les permita conocer el comportamiento de la gestión de acuerdo con la planificación establecida, y posteriormente enlazar a todas las áreas y departamentos en un fin común: medir el resultado de la gestión respecto al cumplimiento de unos objetivos previamente fijados. Asimismo, se detectaron algunos indicadores que reflejan una inadecuada medición estratégica de la gestión, es de ahí que nace la imperante necesidad de que las líneas gerenciales de dichas gerencias migren de la simple medición operativa de gestión a la medición estratégica.

Cabe destacar que la verdadera gerencia, no solo debe cambiar sus modelos mentales y actividades por pensamientos orientados a la creacion de valor, sino diseñar una cadena de gestión que permita satisfacer y deleitar a los clientes objetivos. Por ello, dichas gerencias deberán considerar el implantar mediciones de los resultados, además, deben tomar en cuenta los factores externos que inciden de manera directa en el cumplimiento de las actividades realizadas a través de los procesos.

A partir de los aspectos antes mencionados, se hace notoria la necesidad de identificar los elementos de la planificación estratégica que intervienen en el sistema de medición de gestión de las gerencias mencionadas, las cuales deben considerar la adopción de nuevas estrategias de medición y control, con el propósito de obtener el mejor balance de medición, desempeño y control de sus actividades; a fin de cumplir con los estándares exigidos por clientes, directivos y entorno organizacional, de modo de poder impulsar el uso efectivo de los recursos materiales, humanos y tiempo a utilizar, de manera que permita prever, analizar y corregir problemas que puedan afectar la producción.

Bajo esta óptica, se planteó la siguiente interrogante: ¿Cuáles son los elementos de la planificación estratégica presentes en el sistema de 
medición de gestión de las gerencias de planificación y gestión de la industria petrolera venezolana en la región occidente?

\section{Sistema de medición de gestión}

El diseño de un modelo de medición de gestión debe iniciarse teniendo claridad sobre la estrategia de la empresa, sus factores claves de éxito, sus competencias básicas, sus objetivos y las estrategias definidas por la organización para el logro de su misión y visión (Serna, 2008). Para este autor, los índices miden los logros alcanzados en la ejecución de las estrategias frente a los factores clave del éxito en el mercado.

El sistema de medición de la gestión, a criterio de Cortés y Usme (2004), permite la interacción entre las etapas del ciclo administrativo: planeación, organización, dirección y control, la cual sustenta la elaboración de planes de mejoramiento, de donde se debe obtener como resultado altos niveles de calidad, eficiencia y eficacia. De manera más específica, Serna (2008) define el sistema de medición de gestión como el conjunto de indicadores medibles derivados del plan estratégico que permite evaluar mediante índices el alineamiento entre estrategias, objetivos, acciones, y resultados, por tanto, determinar el desempeño de la organización frente a su direccionamiento estratégico.

En este contexto, una de las funciones del sistema de medición de gestión es sentar las bases sobre las cuales se propongan planes que incidan en el mejoramiento continuo organizacional; garantizar que el sistema de control disponga de mecanismos propios de verificación y evaluación que garanticen objetividad y seguimiento a la gestión. Así, es importante tener claro algunos términos básicos relacionados al sistema de medición de gestión, entre ellos: gestión, medición y mejoramiento continuo organizacional.

Desde la óptica planteada, puede decirse que la gestión generalmente está asociada a las acciones y decisiones de los ejecutivos superiores en una organización. En este caso, es preferible entender la gestión de una manera más genérica y asimilarle a la respuesta que normalmente da un funcionario de una organización, a los requerimientos que le demanda esa misma organización, en atención a sus aptitudes, conocimientos, experiencia, compromiso, esfuerzo y motivación.

En cuanto a la medición, este es un concepto que atraviesa transversalmente a toda la organización, según manifiestan Cortés y Usme (2004), de esta manera se diseñan los indicadores de gestión que permitirán comparar los resultados obtenidos con los estándares establecidos y encender las respectivas alarmas en el momento oportuno, lo que se transforma en planes de mejoramiento, en caso de no ir avanzando hacia el logro de las metas propuestas.

Uno de los procesos que asegura una eficiente medición de la gestión lo constituye el control de gestión, el cual a juicio de Skinner (2010), ha ido evolucionando con el tiempo, a medida que la problemática organizacional planteaba nuevas necesidades y exigencias. Hoy se puede diferenciar un enfoque clásico, sobre el que existe relativo consenso y un nuevo enfoque atomizado en distintas interpretaciones.

Desde el punto de vista de las dimensiones organizacionales, el control de gestión va más allá del ámbito financiero, relacionándose con variables no económicas, que son la base del resultado del negocio (diversificación de los mercados, desarrollo tecnológico, acortamiento de los ciclos de vida de los productos, la calidad, el plazo de entrega y el servicio al cliente, entre otras). En consecuencia, el control de gestión puede ser considerado en un plano cuyas aristas se mueven en tres dimensiones: estratégica, operativa y económica (Figura 1) y tiene como núcleo el sistema informativo (S.I.). 
Figura 1. Dimensiones del control de gestión.

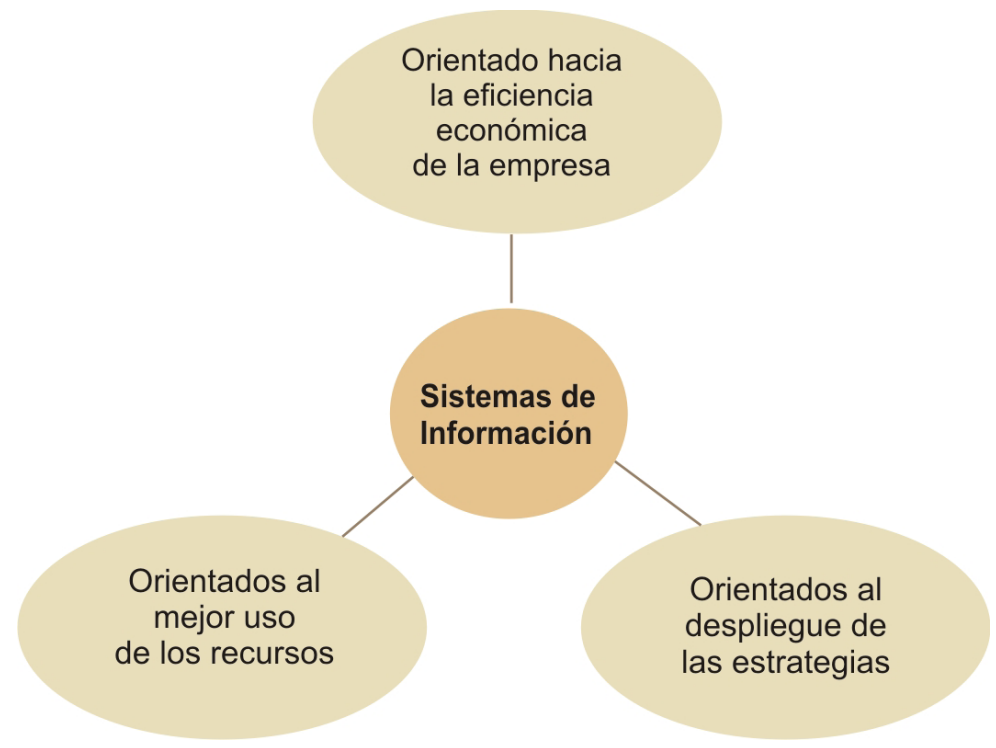

Fuente: Adaptado de Serna (2008)

De esta manera, a través del control de gestión, la empresa configura sus decisiones estratégicas mediante el análisis del entorno y las posibilidades, junto a los potenciales propios de la organización; evalúa la implementación de las decisiones de forma operativa, verificando el cumplimiento de los procedimientos y procesos, y realiza los análisis económicos sobre la base de un sistema informativo eficiente, oportuno y eficaz, que permita la corrección de las desviaciones y su seguimiento.

En esta perspectiva, para los investigadores, los sistemas de medición de gestión buscan generar confianza en las partes interesadas, sobre las actividades de la organización. Para lograr esto, es importante contar con mecanismos de medición que retroalimenten a la organización y que permitan mejorar la toma de decisiones, así como la mejora de sus procesos. Se debería identificar y diseñar indicadores, como parte de la planeación estratégica y de la planeación de los procesos, de manera que se obtengan mediciones adecuadas del desempeño de los objetivos estratégicos y de los procesos.

\section{Elementos de la planificación estratégica}

Desde el punto de vista de las teorías administrativas, las organizaciones, en general, buscan alcanzar determinados fines a partir del trabajo coordinado entre personas, usando medios disponibles. Independientemente de su tipo, poseen tres características que las distinguen: son creadas de manera intencional y de acuerdo con un plan (la mayoría de las veces) para alcanzar ciertas metas; poseen una estructura y orden de carácter vinculante; $y$ con la ayuda de este orden o estructura se puede coordinar la acción de los integrantes y de los recursos disponibles, a fin de cumplir las metas.

Esto es algo simple de entender en abstracto, pero cuando se analizan las percepciones de los participantes en los trabajos de una organización esto puede ser muy complejo. En este sentido, se hace complejo porque estas organizaciones son formaciones sociales, con múltiples influencias y procesos evolutivos diferenciados, a los que concurren variados intereses. La dificultad estriba en que hay que lograr consensos, definiciones y actividades que comprometan a sus integrantes organizacionalmente en cosas de las que no están totalmente convencidos todos.

En este marco de referencia, para orientar sus acciones la teoría administrativa dice que las organizaciones requieren definir de forma explícita 
los fundamentos de su existencia, expresada como la misión institucional. Lo mismo vale para los objetivos y el período de tiempo en que se ha propuesto lograrlos (Francés, 2006). Así, la planificación estratégica cumple dos roles: contribuir a definir el horizonte de desarrollo de la organización $y$, permitir realizar alineamientos entre las distintas unidades que la componen; es decir, fijar orientaciones y metas comunes para la acción.

Desde esta óptica, según Serna (2008), la planificación estratégica es una herramienta de negocios que se puede utilizar para formular estrategias sobrela base delas fortalezas, debilidades, amenazas y oportunidades de las organizaciones, donde las fuerzas externas, tales como las personas, las regulaciones gubernamentales, los cambios en las necesidades de los clientes, los avances tecnológicos y la evolución de la economía ponen a prueba el talento empresarial y la capacidad de reacción ante la presencia de estas fuerzas.

Hoy, considera Amaya (2007), se reconoce el carácter complejo en el mundo de los negocios, por tanto, la planificación estratégica es una herramienta de primer orden en la administración, antes de tomar decisiones sobre la organización, ejecución y control de los planes, programas y proyectos de inversión que adelantan las empresas modernas, por tanto constituye un proceso dentro de las ciencias administrativas que tiene como finalidad mejorar la capacidad de respuesta ante los cambios del entorno.

De esta forma, citando a Corredor (2007), las experiencias demuestran el hecho de que las organizaciones que mantienen una buena planificación a largo plazo se desarrollan mejor en un mercado cambiante; por esta razón la planificación estratégica ayuda a la gerencia a determinar donde debería estar la organización en los próximos años e identificar los recursos que necesita para llegar allá.

Ahora bien, para Serna (2008), el proceso de planificación estratégica considera una serie de elementos, cuya interrelación permite lograr la eficiencia organizacional de la misma. Así, según el autor, la planeación estratégica se compone de seis elementos claves: estrategas, diagnóstico estratégico, direccionamiento estratégico, formulación estratégica, operacionalización e implementación estratégicas. En la Figura 2 se muestra la interrelación de estos elementos desde la óptica del autor citado.

Figura 2. Modelo de planificación estratégica.

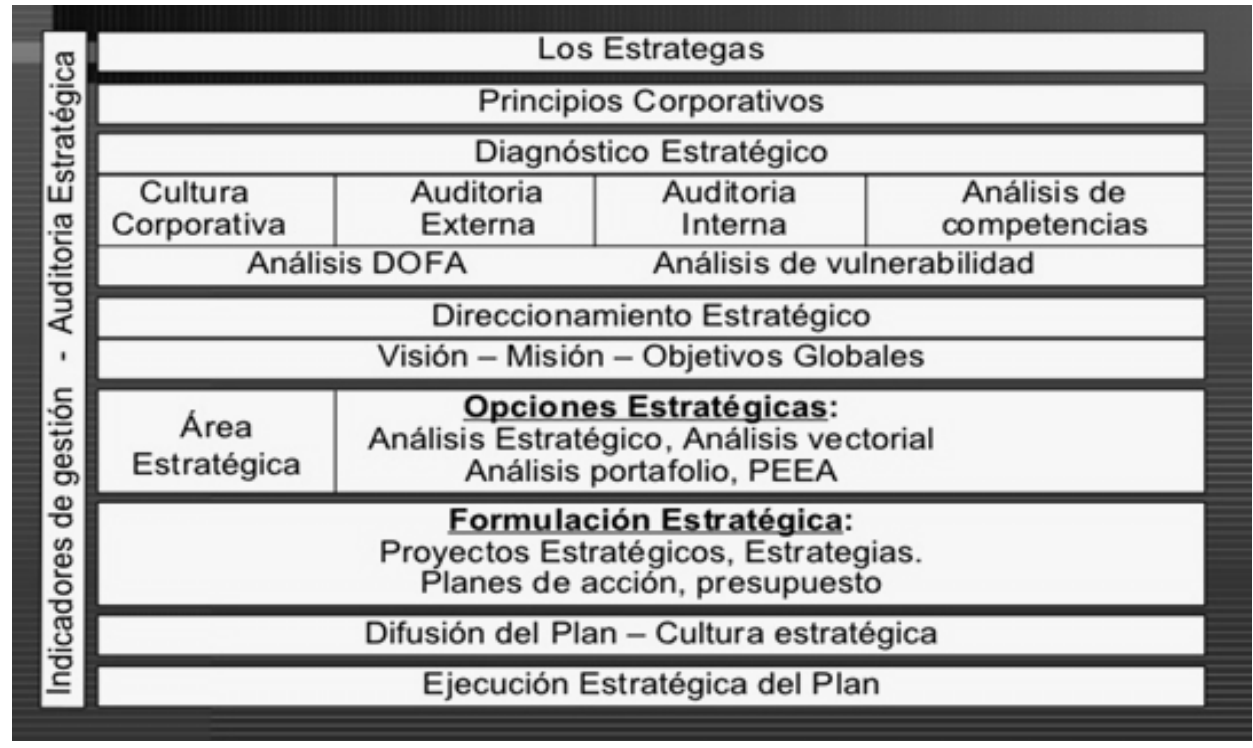

Fuente: Serna (2008) 
Con base a lo referenciado, a juicio de los investigadores, la planificación estratégica es un proceso fundamental que nutre de información pertinente al momento de establecer el sistema de medición de gestión. Por ello, se asumen como elementos de la planificación estratégica los reseñados por Serna (2008): estrategas, direccionamiento, diagnóstico, opciones, formulación, índices de gestión, difusión y alineación estratégicas; como puntos de referencia para analizar el sistema de medición de gestión en las gerencias.

Refiere el autor que los estrategas son aquellas personas ubicadas en la alta dirección de la empresa (miembros de la junta directiva, presidente o gerente y vicepresidentes), a quienes corresponde la definición de objetivos y políticas de la organización.

Por su parte, para David (2010), el direccionamiento estratégico constituye los principios de la organización, que proyecta la imagen del futuro que se decide crear e incluye definir objetivos en diferentes niveles, cuyo alcance va desde lo universal hasta lo particular. A su vez, Wayne (2005) explica que un diagnóstico estratégico se deriva de la selección, concentración y análisis de los factores que afectan a la empresa, en su interior y en su entorno.

Luego de definido el direccionamiento estratégico, realizado el diagnóstico estratégico y el análisis DOFA, deberán explorarse las opciones que la empresa tiene para anticipar tanto sus oportunidades y amenazas, como sus fortalezas y debilidades (Serna, 2008).

Ahora bien, según Goodstein, Nolan y Pfeiffer (2006), la formulación estratégica, es la parte de la planificación estratégica, donde se realiza el proceso de análisis que permite la selección de las áreas en las que la gerencia desea proyectar sus esfuerzos, a través de la información obtenida del direccionamiento estratégico. Dicen los autores que es a través de la formulación estratégica, que la gerencia define los proyectos por medio de los cuales se pretende cumplir con la misión y así lograr la visión.
Dado que, el modelo de planificación estratégica que se aborda, parte de los criterios teóricos de Serna (2008), el mismo parte del supuesto de que el proceso estratégico es una tarea distribuida en cascada entre los niveles de la organización, de arriba hacia abajo y viceversa. Se inicia en el nivel superior de la organización (planeación corporativa), en el cual se definen: objetivos corporativos, valores, visión, misión y estrategias globales de la organización. Representa la planeación macro, a largo plazo, mucho más estable y con una participación limitada de los niveles superiores de la empresa.

Como complemento de lo anterior, Serna (2008) también hace énfasis en que los índices de gestión resultan ser una manifestación de los objetivos estratégicos de una organización a partir de su misión. Igualmente, resultan de la necesidad de asegurar la integración entre los resultados operacionales y estratégicos.

Finalmente, en este marco de referencia, Amaya (2007) asegura que el proceso de difusión estratégica es un elemento fundamental de la planificación estratégica, para asegurar la real y efectiva ejecución de la estrategia de una organización.

\section{MÉTODO}

El tipo de investigación realizada en este trabajo, de acuerdo a la información que se poseía y al nivel de análisis que se hizo, es un estudio de carácter descriptivo, con diseño no experimental, transeccional y de campo. Los datos fueron recolectados de una población finita, conformada por las 5 gerencias de planificación y gestión de la industria petrolera venezolana en la región occidente.

Los sujetos informantes fueron directamente el personal calificado de gerentes y líderes, representado por un total de 11 personas a quienes se le aplicó una encuesta, a través de un cuestionario constituido por 54 ítems; con un escalamiento de frecuencia, de cinco alternativas de respuesta: siempre, casi siempre, algunas veces, casi nunca y nunca. 
Para ser aplicado el instrumento, fue necesario establecer la validez de contenido a través del juicio de expertos. Los expertos con experiencia práctico-teórica permitieron asegurar que la variable de interés era realmente evaluada a través del cuestionario, emitiendo comentarios y apreciaciones en cuanto a la pertinencia y a la formulación de los ítems. En relación con la confiabilidad, se utilizó el coeficiente Alfa de Cronbach, cuyo resultado fue de 0,91 , lo cual indicó que el instrumento poseía muy alta confiabilidad, siendo apto para su aplicación.

\section{RESULTADOS}

El análisis de los resultados se realizó basándose en la estadística descriptiva, a través de las medias aritméticas. Esta ha sido estructurada por medio de tablas contentivas de toda la información recogida sobre la variable en estudio. En este sentido, en los apartados siguientes se presentan los datos obtenidos del cuestionario aplicado a la población en estudio, así como también, su análisis y discusión, en relación con las teorías manejadas.

En la Tabla 1 se presenta el resumen de los elementos de la planificación estratégica, con media de 4,14 reflejando, de acuerdo con los encuestados, alta presencia de los elementos de la planificación estratégica en el sistema de medición de gestión de las gerencias de planificación y gestión de la industria petrolera venezolana en la región occidente. La situación es explicada por el comportamiento mostrado por cada uno de los elementos considerados fundamentales en el proceso de planificación estratégica, estudiados en esta investigación.

Tabla 1. Elementos de la planificación estratégica.

\begin{tabular}{|c|c|c|c|}
\hline INDICADORES & $\bar{X}$ & S & CATEGORÍAS \\
\hline Los estrategas & 4,12 & 1,20 & $\begin{array}{c}\text { Alta presencia } \\
\text { Moderada dispersión /Moderada confiabilidad }\end{array}$ \\
\hline Direccionamiento estratégico & 4,27 & 0,95 & $\begin{array}{c}\text { Muy alta presencia } \\
\text { Baja dispersión/Alta confiabilidad }\end{array}$ \\
\hline Diagnóstico estratégico & 4,06 & 0,72 & $\begin{array}{c}\text { Alta presencia } \\
\text { Baja dispersión/Alta confiabilidad }\end{array}$ \\
\hline Opciones estratégicas & 4,15 & 0,95 & $\begin{array}{c}\text { Alta presencia } \\
\text { Baja dispersión /Alta confiabilidad }\end{array}$ \\
\hline Formulación estratégica & 4,41 & 1,12 & $\begin{array}{c}\text { Muy alta presencia } \\
\text { Moderada dispersión/Moderada confiabilidad }\end{array}$ \\
\hline Planeación estratégica en cascada & 3,94 & 1,03 & $\begin{array}{l}\text { Alta presencia } \\
\text { Moderada dispersión/Moderada confiabilidad }\end{array}$ \\
\hline Índices de gestión & 4,12 & 1,04 & $\begin{array}{c}\text { Alta presencia } \\
\text { Moderada dispersión/Moderada confiabilidad }\end{array}$ \\
\hline Difusión y alineación estratégicas & 4,02 & 1,23 & $\begin{array}{c}\text { Alta presencia } \\
\text { Moderada dispersión/Moderada confiabilidad }\end{array}$ \\
\hline RESUMEN DIMENSIÓN & 4,14 & 1,03 & $\begin{array}{c}\text { Alta presencia } \\
\text { Moderada dispersión } \\
\text { Moderada confiabilidad }\end{array}$ \\
\hline
\end{tabular}


De manera detallada, se muestra que en estas gerencias analizadas se realiza con alta frecuencia la línea gerencial como parte integral de los estrategas; la alta dirección promueve el desarrollo de estrategas, vistos como elemento clave del proceso de planificación estratégica; y en la medición de la gestión participan los estrategas que elaboraron el plan estratégico.

De acuerdo con los resultados expuestos para el indicador los estrategas, se logra validar lo expuesto por Serna (2008), para quien los estrategas son aquellas personas o funcionarios ubicados en la alta dirección de la empresa (miembros de la junta directiva, presidente o gerente y vicepresidentes) a quienes corresponde la definición de objetivos y políticas de la organización.

Así las cosas, en las gerencias analizadas para el sistema de medición de la gestión es fundamental conocer el perfil de los estrategas, constituyéndose en un elemento clave dentro del proceso de planificación estratégica, asignándole a ésta la función que tiene por objetivo, fijar el curso concreto de acción que ha de seguirse, estableciendo los principios que habrán de orientarlo, la secuencia de operaciones para realizarlo y las determinaciones de tiempo y números necesarios para realizarlos.

En relación con el direccionamiento estratégico, se evidencia que existe muy alta presencia de los principios de la organización, validando lo expresado por David (2010), para quien la imagen de futuro que se decide crear mediante el direccionamiento estratégico incluye definir objetivos en diferentes niveles, cuyo alcance va desde lo universal hasta lo particular.

De manera que, en las gerencias analizadas, el direccionamiento estratégico es un elemento de la planificación estratégica para su adecuado desarrollo, y está orientado a la formulación de principios, misión, visión, políticas y objetivos. Este aspecto se convierte en una guía fundamental para el sistema de medición de la gestión, al momento de evaluar resultados esperados con lo formulado en el direccionamiento estratégico.
Siguiendo con la secuencia de los resultados, se tiene lo obtenido para el indicador diagnóstico estratégico, donde se observó alta presencia en la elaboración del plan estratégico para el análisis de los problemas. Estos resultados son análogos a los postulados de Wayne (2005), cuando explica que un diagnóstico estratégico se deriva de la selección, concentración y análisis de los factores que afectan a la empresa, en su interior y en su entorno.

Según estas evidencias, las gerencias analizadas identifican los factores internos y externos, para enfrentar y minimizar los efectos negativos $o$ utilizarlos para maximizar sus efectos, los cuales, a su vez, se constituyen en información relevante para nutrir el sistema de medición de la gestión.

En lo que concierne al indicador opciones estratégicas, en las gerencias estudiadas, se realiza con alta frecuencia lo referido a que en el plan estratégico se analice el comportamiento de su portafolio de productos, y determinan las estrategias globales que le permitirán lograr eficientemente su misión.

Los resultados demuestran alta presencia de lo enfocado por Serna (2008), quien afirma que luego de definido el direccionamiento estratégico, realizado el diagnóstico estratégico, el análisis interno y externo, deberán explorarse las opciones que se tienen para anticipar tanto oportunidades y amenazas, como fortalezas y debilidades.

De esta forma, en las gerencias analizadas, las opciones estratégicas surgen del análisis interno y externo en conjunto con otras técnicas de jerarquización; éstas son las posibles rutas o caminos para seguir para alcanzar su visión. Como tal se constituye en un elemento clave dentro de la planificación estratégica, que servirá de guía al momento de que la organización implemente el sistema de medición de la gestión, será entonces un indicador de lo logrado en virtud de lo esperado.

Siguiendo con la secuencia de presentación de los resultados, se alcanzó muy alta presencia de las actividades involucradas a la formulación 
estratégica, resultando análogas a los postulados teóricos de Goodstein et al. (2006), para quienes la formulación estratégica, es la parte de la planificación estratégica, donde se realiza el proceso de análisis que permite la selección de las áreas en las que la gerencia desea proyectar sus esfuerzos, a través de la información obtenida del direccionamiento estratégico.

Por tanto, se deduce que, en las gerencias analizadas, la formulación estratégica es un proceso de la planificación donde se seleccionan los proyectos o áreas estratégicas que han de integrar el plan estratégico, con base a lo acordado en el direccionamiento.

Con relación al indicador planeación estratégica en cascada, se observa que, con muy alta frecuencia, en estas gerencias, el proceso estratégico es una tarea distribuida de arriba hacia abajo y viceversa entre los niveles de la organización. De acuerdo con los resultados expuestos, estas gerencias muestran congruencia con lo planteado por Serna (2008), para quien el modelo de planificación estratégica parte del supuesto de que el proceso estratégico es una tarea distribuida en cascada entre los niveles de la organización, de arriba hacia abajo y viceversa.

Así las cosas, las gerencias bajo estudio se integran en un sistema de interrelaciones que hace de la planificación estratégica un proceso dinámico, ágil, flexible y ampliamente participativo.

De igual manera, se evidenció alta presencia de los índices de gestión como elemento de la planificación estratégica, indicativo que: en las gerencias bajo estudio se presenta con alta frecuencia el hecho de que en el plan estratégico se definan los índices que permiten medir el desempeño de la organización; la auditoría estratégica constituye el elemento fundamental en la consolidación de la cultura estratégica y; los índices de gestión aseguran la integración entre los resultados operacionales y estratégicos.

Estos resultados logran validar a Serna (2008), quien opina que el desempeño de la organización debe monitorearse y auditarse. Para ello, con base a los objetivos, los planes de acción y el presupuesto estratégico, se definirán unos índices que permitirán medir el desempeño de la organización. Estos índices se constituyen en medidas del desempeño de las variables que componen el sistema de medición de la gestión.

De allí que, en las gerencias analizadas, los índices de gestión son una forma clave de retroalimentar sus procesos, de monitorear el avance o la ejecución de los planes estratégicos; constituyéndose en la relación cuantitativa entre las metas planeadas, los objetivos, los estándares relacionados con los indicadores y los resultados logrados.

Ahora bien, los hallazgos del último indicador aplicado en la dimensión que se analiza, denominado difusión y alineación estratégicas, se evidencia alta frecuencia de la difusión del plan estratégico en cascada entre los niveles de la organización, de arriba hacia abajo y viceversa; y la misma se realiza de manera que cada entidad identifique las acciones con que cada uno de ellos va a contribuir al logro de los objetivos particulares.

Estos resultados alcanzan alta coincidencia con lo expuesto por Amaya (2007), quien asegura que el proceso de difusión estratégica es un elemento fundamental de la planificación estratégica, para asegurar la real y efectiva ejecución de la estrategia de una organización. De manera que, en las gerencias analizadas desarrollan un efectivo proceso de difusión y alineación estratégica que facilita el control de la estrategia, en el cual se pueden evidenciar procesos asociados a las perspectivas y objetivos estratégicos, e indicadores asociados.

En términos generales, al ver los resultados de los elementos de la planificación estratégica se evidencia una alta congruencia con lo planteado por Amaya (2007), quien afirma que se reconoce el carácter complejo en el mundo de los negocios, por tanto, la planificación estratégica es una herramienta de primer orden en la administración, 
antes de tomar decisiones sobre la organización, ejecución y control de los planes, programas y proyectos de inversión que adelantan las empresas modernas, por tanto constituye un proceso dentro de las ciencias administrativas que tiene como finalidad mejorar la capacidad de respuesta ante los cambios del entorno.

Así las cosas, en las gerencias analizadas, la planificación estratégica es un proceso fundamental que nutre de información pertinente al momento de establecer el sistema de medición de gestión. En este proceso se asumen como elementos básicos: los estrategas, direccionamiento, diagnóstico, opciones, formulación, índices de gestión, difusión y alineación estratégicas.

\section{CONCLUSIONES}

Una vez desarrollado el procedimiento de la investigación, mediante la fundamentación teórica la recopilación, así como el análisis de los datos, los resultados obtenidos, su interpretación y contraste, se identificaron los elementos de la planificación estratégica con alta presencia en el sistema de medición de gestión. De forma tal que se evidenció muy alta presencia del direccionamiento estratégico y la formulación estratégica. Los elementos: estrategas, diagnóstico estratégico, opciones estratégicas, planeación estratégica en cascada, índices de gestión y difusión y alineación estratégicas, mostraron altas presencias.

Es necesario destacar que, si bien se obtuvo alta presencia de los elementos de la planificación estratégica en el sistema de medición de gestión, siendo esto positivo para las gerencias estudiadas, aun no llega al estándar establecido como máximo en el baremo diseñado, cuya situación ideal sería de muy alta presencia, por lo cual se observan posibilidades de mejora.

La idea debe ser consolidar la aplicación en conjunto de estos elementos, en virtud de que cada uno de ellos está interrelacionado, considerando siempre que, para el sistema de medición de gestión, el proceso de planificación estratégica interrelaciona estos elementos, lo cual permite lograr la eficiencia organizacional del sistema de medición.

\section{REFERENCIAS}

Amaya, J. (2007). Gerencia, planeación y estrategia. Fundamentos, modelo y software de planeación. Colombia: Editorial SYC Publiarte

Corredor, J. (2007). La planificación estratégica. Venezuela: Vadell Hermanos Editores.

Cortes, A. y Usme, M. (2004). Diseño e implementación de un sistema integral de medición de gestión (SIMG). Revista Scientia et Technica, X(24): 183-188. DOI: http://dx.doi. org/10.22517/23447214.7341

David, F. (2010). Gestión Estratégica: Conceptos y Casos. México: Décima tercera edición. Pearson Prentice Hall

Francés, A. (2006). Estrategias y planes para la empresa, con el cuadro de mando integral. México: Pearson educación

Goodstein, L., Nolan, T. y Pfeiffer, J. (2006). Planeación estratégica aplicada. Primera Edición. Santa Fe de Bogotá-Colombia: Editorial Mc Graw Hill

López, S. (2008). La efectividad gerencial. Venezuela: Editorial Casa Hermanos

Serna, H. (2008). Gerencia Estratégica. Teoría, Metodología. Alineamiento, Implementación y Mapas Estratégicos, Índices de Gestión. Décima edición. Bogotá-Colombia: 3R Editores

Skinner, W. (2010). Administración estratégica de operaciones. México: Mc Graw Hill

Terán, F. (2011). La prevención de pérdidas y su relación con el manejo financiero del riesgo. VIII jornadas de control de riesgos. Petróleos de Venezuela S.A. Venezuela

Wayne W. (2005). Planeación estratégica aplicada. México: Editorial Pearson 